\title{
Rapid prototyping of reflectors for vehicle lighting using laser activated remote phosphor
}

\author{
Roland Lachmayer, Gerolf Kloppenburg* and Alexander Wolf \\ Leibniz University Hannover, Institute of Product Development, Welfengarten 1A, \\ 30167 Hannover, Germany
}

\begin{abstract}
Bright white light sources are of significant importance for automotive front lighting systems. Today's upper class vehicles mainly use HID or LED as light source. As a further step in this development laser diode based systems offer high luminance, efficiency and allow the realization of new styling concepts and new dynamic lighting functions. These white laser diode systems can either be realized by mixing different spectral sources or by combining diodes with specific phosphors.

Based on the approach of generating light using a laser and remote phosphor, lighting modules are manufactured. Four blue laser diodes $(450 \mathrm{~nm})$ are used to activate a phosphor coating and thus to achieve white light. A segmented paraboloid reflector generates the desired light distribution for an additional car headlamp. We use high speed milling and selective laser melting to build the reflector system for this lighting module. We compare the spectral reflection grade of these materials. Furthermore the generated modules are analyzed regarding their efficiency and light distribution. The use of Rapid Prototyping technologies allows an early validation of the chosen concept and is supposed to reduce cost and time in the product development process significantly. Therefor we discuss costs and times of the applied manufacturing technologies.
\end{abstract}

Keywords: Automotive Lighting, Laser Activated Remote Phosphor, Laser Light Source, Rapid Prototyping of Optical Components, Selective Laser Melting

\section{INTRODUCTION}

\subsection{Automotive Headlamps}

From the beginning of this century, development in general lighting has moved from the use of incandescent lamps as a light source towards semiconductor based lights such as LEDs. This also applies to the automotive sector where more and more LED based headlamps have been presented lately. Due to an increase in luminous flux and total system efficiency of single LEDs as well as LED arrays, today's LED headlamps can provide enough light for a complete high beam and low beam distribution. Using semiconductors with a small package size and a small area of light emission, new possibilities for headlamp design arise, for example regarding a reduced headlamp height.

\subsection{White Light with Laser Diodes}

As a further step in this development, the use of laser diodes is evaluated for general lighting applications. Laser diodes are also semiconductor light sources as LEDs. The light is generated by electrically stimulating a doped semiconductor material like InGaN. Resulting from this, the material emits photons of a certain energy level and thus a certain narrow range of wavelengths. To generate white light from this spectrum, the same functional principle as for most white LEDs is used consisting of a blue source $\left(450 \mathrm{~nm}\right.$ ) and a phosphor ${ }^{1}$ (as shown in figure 1-a). In contrast to white LEDs, the phosphor for laser activated white light is in a remote position from the energy emitting semiconductor chip so that the concept is often called remote phosphor. The separation of light generation and conversion is possible due to the directional emission characteristics of laser diodes and thus allows improved cooling concepts for the systems. ${ }^{2}$

An automotive lighting system based on laser activated remote phosphor has been introduced by BMW in their i8 as the first series-production vehicle to use this technology. ${ }^{3}$ Other car manufacturers like AUDI and Daimler have also presented or announced laser based headlamp systems ${ }^{4,5}$.

*E-mail: kloppenburg@ipeg.uni-hannover.de, Telephone: +49 5117623470

Light-Emitting Diodes: Materials, Devices, and Applications for Solid State Lighting XIX, edited by

Klaus P. Streubel, Heonsu Jeon, Li-Wei Tu, Martin Strassburg, Proc. of SPIE Vol. 9383, 938305

(C) 2015 SPIE $\cdot$ CCC code: $0277-786 X / 15 / \$ 18 \cdot$ doi: 10.1117/12.2078791

Proc. of SPIE Vol. $9383938305-1$ 


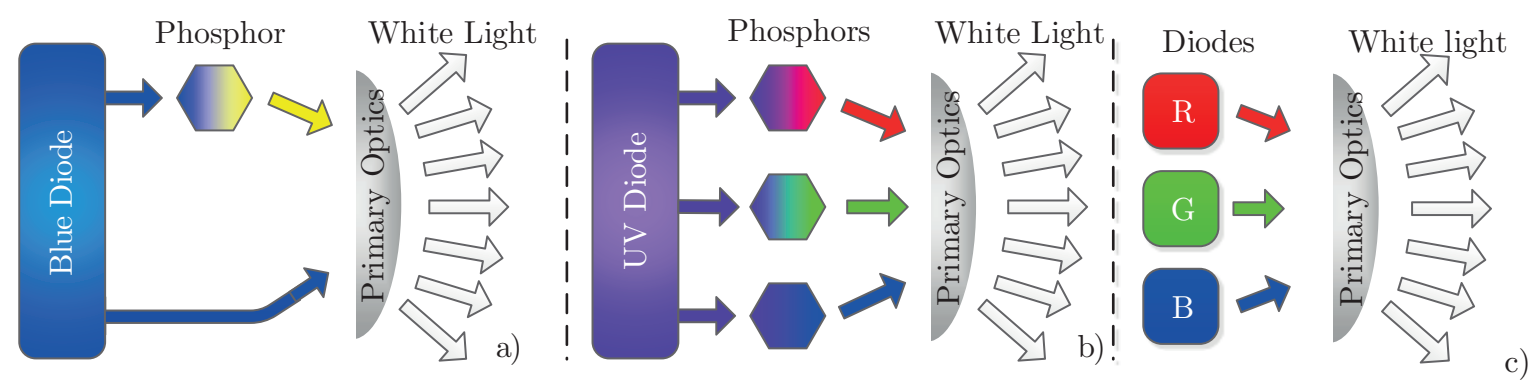

Figure 1. Generation of white light using laser diodes: a) Mixing direct blue light and phosphor-converted light; b) Mixing different phosphors all converting from UV light; c) Mixing diodes with different emission wavelengths ${ }^{6}$

\subsection{Selective Laser Melting}

The selective laser melting process enables the production of complex-shaped geometries which can't be produced with different technologies. One typical example are contour-close cooling-channels for freeform geometries in casting molds. In the SLM process the model is generated in layers. A thin layer of metal powder is being spread onto a base platform. The areas of the powder which shall become part of the model are melted by a laser beam and solidify when cooling down. Then the next layer of powder is disposed on top of the last one. The laser melts the parts of the model in this cross section and the process starts anew.

A common use for the SLM process is the production of prototypes. Even though a realization of the geometries may be possible using 5-axis milling or other manufacturing technologies, the use of SLM offers a great chance to save time in the preprocessing steps.

Focusing the manufacturing of optical components, the SLM technology is suitable to generate bionic cooling structures for the use with LEDs $^{7,8}$, laser diodes or heat emitting phosphor coatings. Furthermore, the manufacturing of prototypes of freeform reflectors is an interesting aspect when using aluminum as material, since no additional reflective coating is required. According to the machine manufacturer, the material density of produced parts is about $99.85 \%$ for aluminum ${ }^{9}$ and $100 \%$ for maraging steel. ${ }^{10}$ A significant porosity would not only reduce the reflectance of a SLM mirror but also leads to undesired stray light. Therefore a good knowledge about the expected amount of pores, the locations of their occurrence and strategies to reduce them is required. Due to the hot production process a thermal distortion of the part will occur, which significantly depends on the part's geometry as well as on its orientation during the building process. Thus, mathematical models have to be developed to forecast these distortions and to avoid them by numerically deforming the CAD part appropriately before the melting process.

In this paper the properties of post-processed laser-sintered surfaces will be investigated. The concept of a static laser-based additional high beam will be realized using rapid prototyping technologies. Focusing on the generation of the main reflector of this automotive headlamp, the laser melting process is compared with 5-axis high-speed milling. The concept of the additional high beam is presented in chapter 2 .

\section{CONCEPT AND REFLECTOR DESIGN}

\subsection{Optical Design}

Various optical concepts are possible for an automotive lighting module. For the reflector based principle used in this case, the position of the light source (meaning the light generating phosphor) inside the reflector is fixed by the reflector design, but there are still various possibilities for the position of the laser diodes and how to guide the light to the phosphor layer. Since lasers are fairly easy to attach to a light guide, one approach can be to completely separate the laser source from the rest of the system and then positioning a light guide in a way the phosphor can be activated. Other solutions include using the laser beam directly to activate a transmissive or reflective phosphor. We focus on the reflective designs which can be grouped into two general options: front and rear projection. Rear projection means, the laser diodes are placed behind the reflector emitting directly towards the phosphor inside. The converted light is then emitted back towards the reflector surface. The chosen 


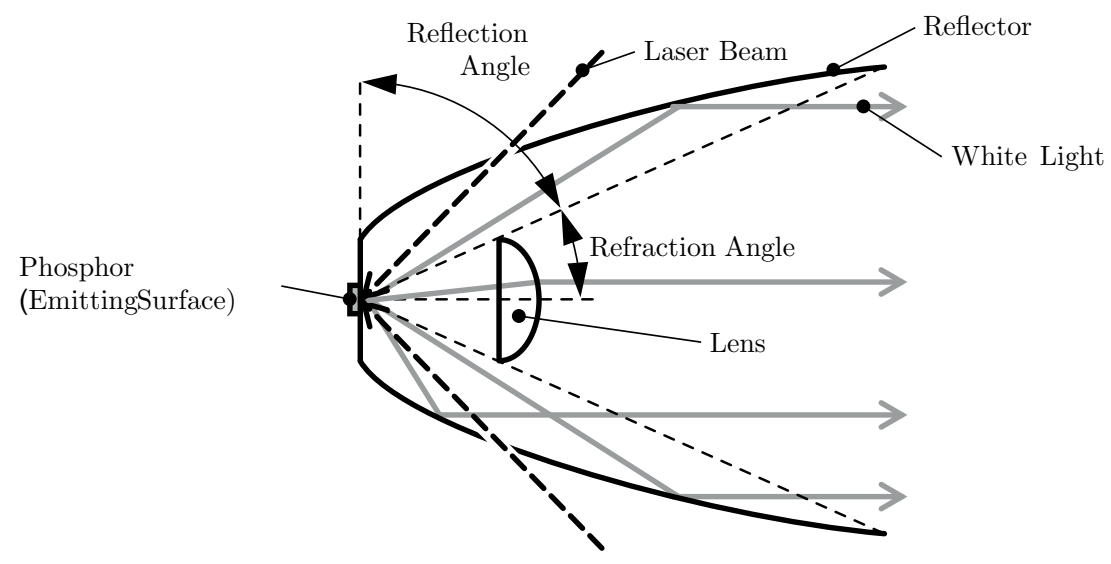

Figure 2. Concept of reflector and light generation. ${ }^{11}$

design is using a front projection where the laser beam enters the system from the front and thus resulting in the phosphor to emit light as a Lambertian source to the front. This allows to use a maximum of the generated white light for the desired light distribution. To avoid a safety risk due to a damaged phosphor layer, in addition to the entrance holes for the laser beam, exit holes on the opposite side have been added. The concept is depicted in figure 2. Since for the principle of front projection not all emitted light hits the reflector surface, a lens has been added to further shape the light in the desired way. This lens has been produced using a high speed cutting machine but will not be regarded any further in this article. The complete module with four laser diodes is shown in figure 3.

In the UN/ECE regulations regarding high beam light for vehicles (regulation $113^{12}$ ) there are also values for the maximum illuminance on a test screen for additional high beam modules. These modules are installed to support the existing high beam and to extend its range (and thus the visibility range). There are no regulations on laser based vehicle lights yet but regarding its light distribution, the presented module should be validated against the values mentioned in regulation 113. Figure 8 shows the light distribution of the investigated prototypes of the reflector measured with a goniophotometer. But the discussion concerning a proper light distribution of additional high beam systems has just started. ${ }^{13}$

\subsection{Thermo-Optical Coupling}

Laser diodes are quite small light sources with regard to their emission surface. Therefore they can nearly be seen as point light sources allowing a significant reduction of the dimensions of illumination systems without reducing

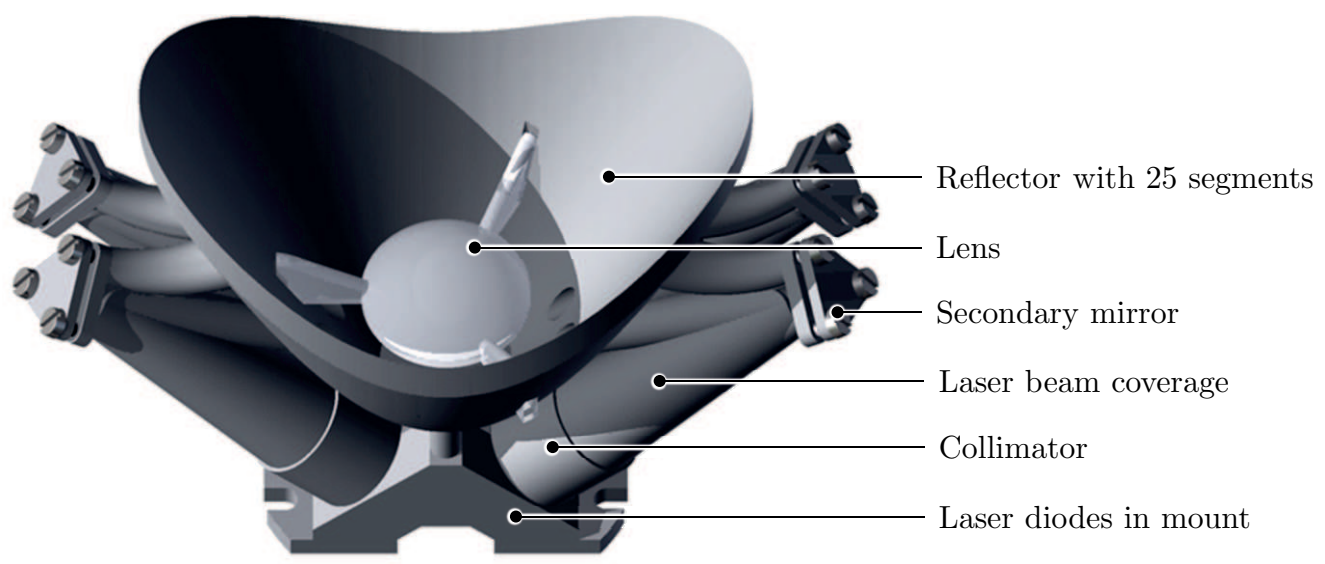

Figure 3. CAD model of the reflector. ${ }^{14}$ 


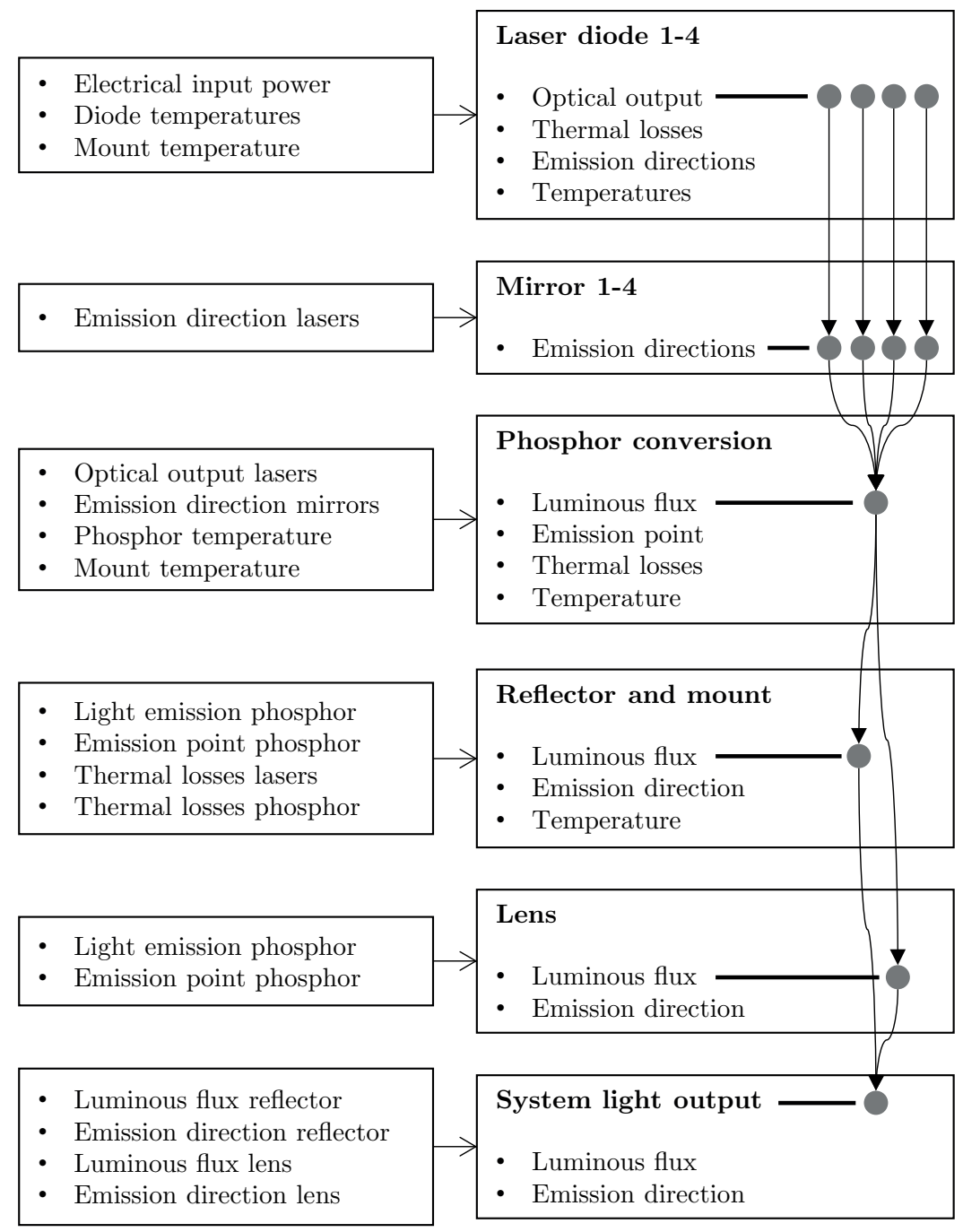

Figure 4. Influence factors of the light generation in the reflector.

their efficiency. This opens up the possibility for new design approaches like extremely flat car headlamps. To make use of this opportunity a very precise adjustment of the optical components is required. Due to thermal losses of laser diodes and conversion layer as well as changed surrounding conditions the dependency of this adjustment from the system's temperature has to be minimized. The most significant parameters affecting the components of the presented optical system are given in figure 4 . The intended path of the light is denoted with arrows and grey dots in the right half of the figure.

The optical output of a laser diode and thus its efficiency strongly depends on the case temperature. This temperature is mainly affected by the thermal losses of the diode itself, the temperature of the diode's mount and the thermal resistance between both. In order to achieve a good cooling the four diodes in the module presented are mounted in a block of solid aluminum. This block is connected with the aluminum reflector and actively cooled. The fixed mechanical connection between diode and block gives a low thermal resistance and hence reduces the case temperature of the diode.

The direction of the laser beam has to be adjusted in order to hit the phosphor conversion layer in the focal point of reflector and lens. This is achieved by adjusting secondary mirrors (one mirror for each diode). The 
mirrors are mounted on additional posts on both sides of the main reflector. On the one hand these additional 'arms' lead to a complex shape of the reflector, on the other hand the connection between reflector and mirrors offers a rigid system with a low thermal resistance. Thus the orientation of the secondary mirrors is only slightly affected by the systems operating conditions.

As for the diodes, the efficacy of the phosphor conversion layer strongly depends on its temperature. This effect is called thermal quenching ${ }^{1,15}$. Due to its thermal losses the conversion layer is self-heated. Therefore a phosphor which is coated on a metal substrate is used in this case. This substrate spreads the generated heat. The metal plate is fixed at the back side of the reflector.

The reflector as well as the lens project the light spot from the conversion layer onto the street. The emission direction of the system depends on the geometrical relations between conversion layer, reflector and lens. This temperature dependency is kept small by using aluminum as reflector material. The lens consists of PMMA so that its exact dimensions may be influenced by the systems temperature.

A more detailed discussion of the influence factors on laser-generated white light can be found in a previous publication. $^{2}$

\section{COMPARISON OF MANUFACTURING METHODS}

In order to produce the reflector introduced in section 2, several technologies may be applicable. In this case the lot size is small so that the rapid prototyping technologies high-speed milling and selective laser melting are taken into account.

Using SLM nearly every geometry seems to be manufacturable. In reality several design rules have be taken into account ${ }^{16,17}$. In order to enable a heat transport from the building part to the platform the part either has to be sintered directly on the platform or connected to it using support structures. Every surface that has an overhang with angles of more than typically $40^{\circ}$ has to be fixed by support structures as well. If a structure is long regarding its dimension in building direction (z-direction) and thin in $\mathrm{x}$ - and y-direction it should either be supported on its side faces or by placing a solid object very close to it. For the example reflector typical times for the support generation, machine preparation as well as the building time and the time to separate the object from the platform and to remove the support structures are given in table 1. Usually the upside faces (pointing in building direction) are of better quality than the downside ones (pointing to the platform). Thus the reflector is placed with the reflectance surface, whose quality is most important, facing upwards. Due to the laser-wise production process surfaces with a small angle towards the horizontal x-y-plane (up to $5^{\circ}$ ) should be avoided. Otherwise they will show significant steplike structures. Since the faces of the reflector are quite steep no problem is expected here. As another design aspect, it is possible to generate thinner walls with aluminum than with many other SLM materials due to its low specific weight and good thermal properties. ${ }^{18}$

Table 1. Comparison of the processing steps for HSC and SLM

\begin{tabular}{cllll}
\hline Step & \multicolumn{2}{l}{ High-speed milling } & \multicolumn{2}{l}{ Selective laser melting } \\
No. & Time $(\mathrm{h})$ & Processing step & Time $(\mathrm{h})$ & Processing step \\
\hline 1 & $*$ & CAD model generation & $*$ & CAD model generation \\
2 & 6.0 & CAD/CAM & 0.5 & Support generation \\
3 & 1.0 & Machine preparation & 3.0 & Machine preparation \\
4 & 6.0 & Milling & $5.5^{* *}$ & Selective laser melting \\
5 & \multicolumn{5}{l}{0.5} & Separation from platform, support removal \\
6 & $* * *$ & Finishing & $* * *$ & Finishing \\
$*$ & Not evaluated & \\
$* *$ & 8 reflectors simultaneously: $14 \mathrm{~h}(1.8 \mathrm{~h}$ per reflector $)$ \\
$* * *$ & To be discussed in section 5.3 \\
\hline
\end{tabular}


The manufacturing times listed up in table 1 strongly depend on the machines and tools used and the knowledge of the operator. Therefore they can only be used as indicators. In our case an imes-icore 4030 $\mu$ premium is used as HSC machine and an EOS M280 as SLM machine. Each layer in the SLM-process has a strength of $30 \mu \mathrm{m}$. Deviating from the EOS reference nitrogen is used as inert gas instead of argon. This is standard for machines from other manufacturers.

The high-speed milling process with five axis also allows the generation of the reflector. Due to its quite complex shape the machining takes part with a lot of different orientations. Hence several points of origin are generated in the $\mathrm{CAD} / \mathrm{CAM}$ system which in consequence leads to a duration of this step of about $6 \mathrm{~h}$.

\section{REFLECTANCE OF THE SLM MATERIAL}

In order to evaluate the reflectance properties of the material used in the SLM process (AlSi10Mg), cylindrical test parts are manufactured. The four examples are produced with a different amount of applied energy at the parts' surfaces. For two of them a high amount of energy is used, the other two samples are produced with energy set to a low level. One of these two parts respectively is placed with its surface normal of the evaluated reflectance surface parallel to the building direction and the other one perpendicular to it.

The cylindrical test parts are polished with a device used to polish micrograph probes. This allows reproduceable results since hand-polishing is extremely difficult with this forgeable alloy.

The test probes are illuminated with a collimated incandescent bulb with an incident angle of the light of $45^{\circ}$. The reflectance is measured depending on the wavelength. The measurements indicate a dependence between the reflectance of the test probes and their manufacturing conditions (figure 5). The two parts DP1 and DP2 offer a relatively good reflection grade and are build with less laser power per area than the samples DP3 and DP4. Evaluating the spectral reflectances of the test parts, it is remarkable that the reflectance is nearly constant against the wavelength although results from other publications give a decreasing reflectance with increasing wavelength for conventional aluminum. Also the reflectance for visible light is much higher, about $90 \%{ }^{19}$ According to the measurements, all four test parts show nearly the same spectral reflection behavior. Hence more measurements are necessary in order to evaluate this effect in detail since the measurement uncertainties are high in this case. The gained spectral reflectance values are weighed according to the $V(\lambda)$-function (table 2). This provides a reflectance value corresponding to the sensitivity of the human eye.

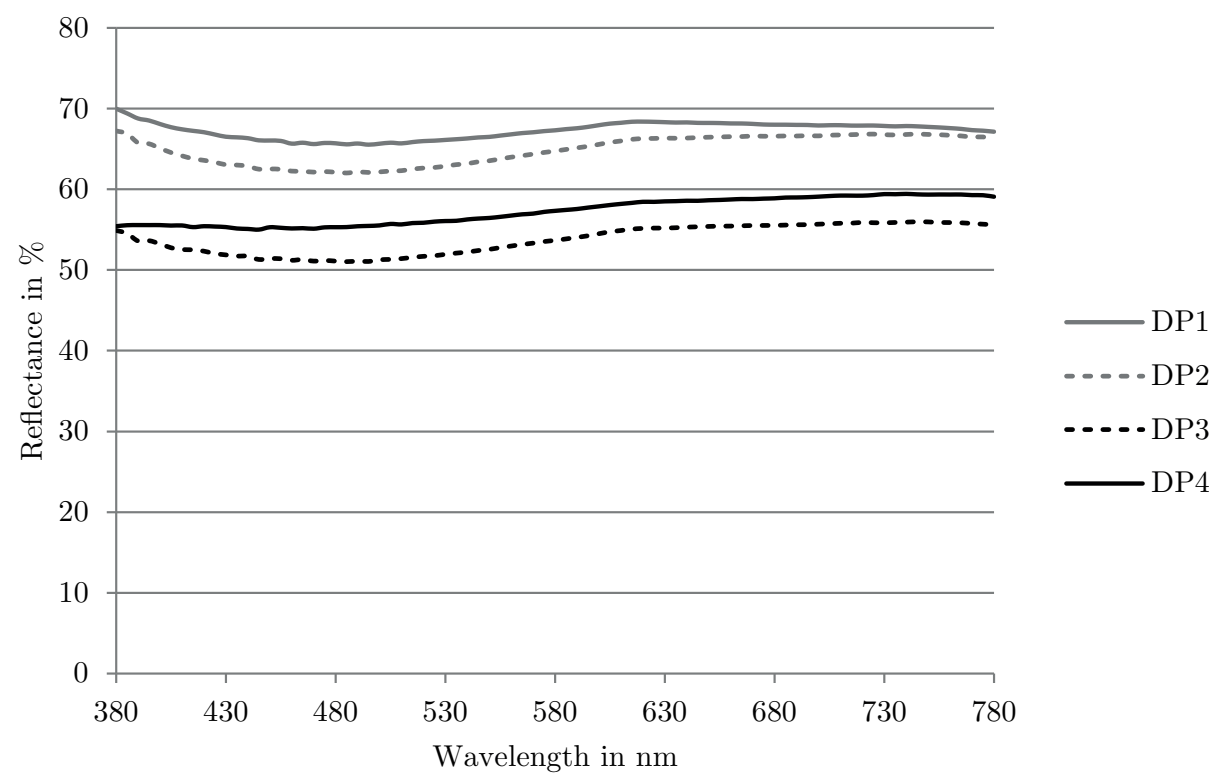

Figure 5. Spectral reflectance of the test parts. 

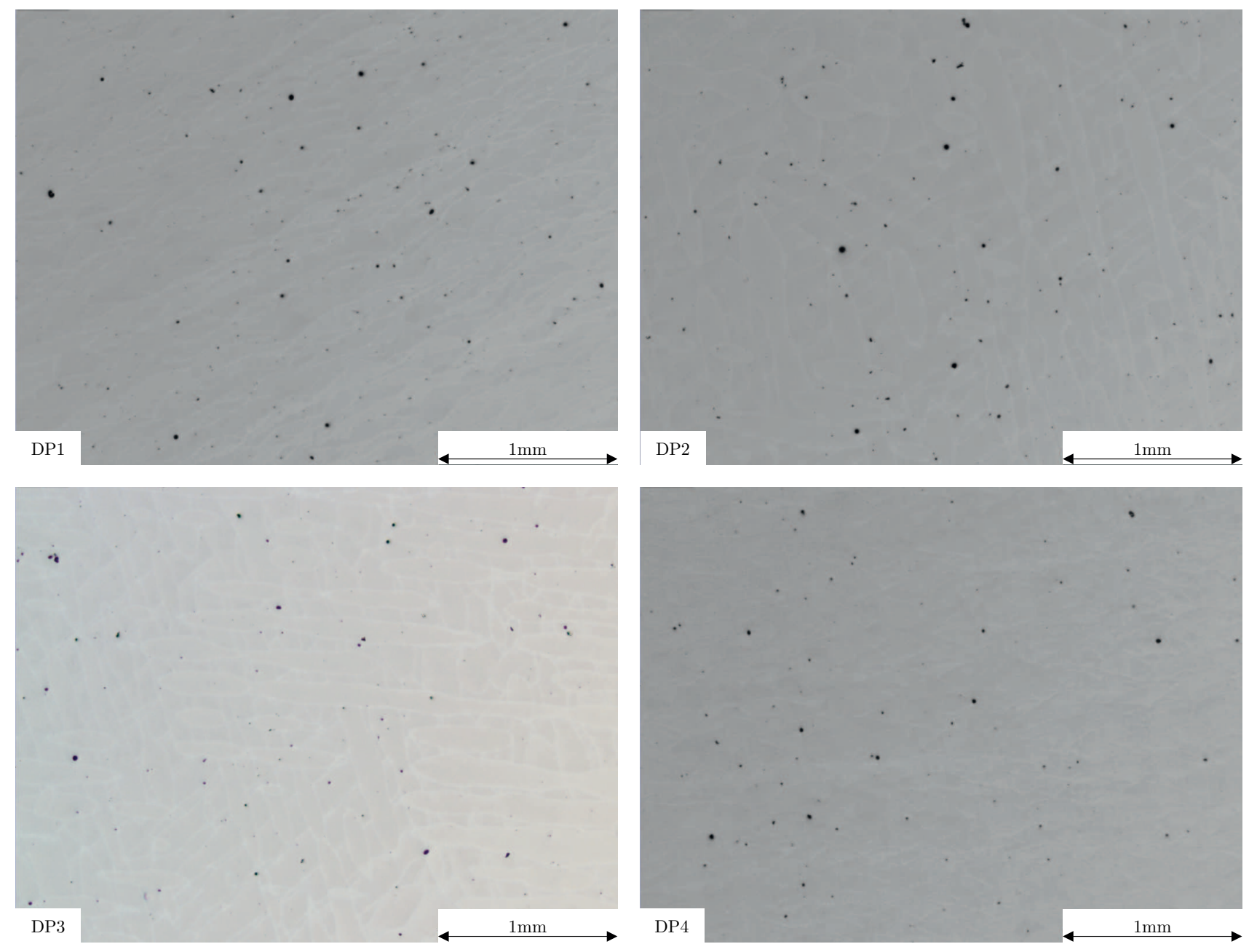

Figure 6. Polished cut images of SLM parts.

The samples DP1 and DP4 may show a dependency between their reflection grade and their peripheral angle because these samples represent cross sections with a strong influence of the layer-wise building process. Nevertheless the measurements were done in an arbitrary peripheral orientation.

It has to be taken into account that without any protective coating the reflection grade of the aluminum will over time be reduced due to oxidation. All measurements were done without any coating, but quite soon after the surface treatment and the samples were covered with an appropriate tape whenever they were not in use.

Table 2. Reflectance and porosity of laser-sintered parts

\begin{tabular}{ccccc}
\hline $\begin{array}{c}\text { Sample } \\
\text { No. }\end{array}$ & $\begin{array}{c}\text { Laser power } \\
\text { per area }\end{array}$ & $\begin{array}{c}\text { Orien- } \\
\text { tation }\end{array}$ & $\begin{array}{c}\text { Reflectance } \\
(\%)\end{array}$ & $\begin{array}{c}\text { Porosity } \\
\text { Area }(\%)\end{array}$ \\
\hline DP1 & Low & Perpendicular & 67 & 0.20 \\
DP2 & Low & Parallel & 64 & 0.24 \\
DP3 & High & Parallel & 53 & 0.16 \\
DP4 & High & Perpendicular & 57 & 0.17 \\
\hline
\end{tabular}


The polished cut images from the test samples (figure 6) are used to calculate their porosity values (table 2). This is done by measuring the amount of dark pixels in the images using the open source software imageJ. According to the increased laser power insertion the porosity of the samples DP3 and DP4 is lower than that of the other parts.

The given values are dependent on the measurement position since the porosities mainly occur close to the outer surface of an object. Since the exact amount of abrasion from the parts is unknown, these values are of qualitative nature.

The cut images also indicate the grain structure of the test samples. Due to the orientation of the parts DP1 and DP4 during the manufacturing process, the cross-sections show several manufacturing layers which can be seen in figure 6 . The grains are significantly noncircular. The other two pictures show through nearly one single layer. Therefore, the grains show a more regular shape in this cross-section.

\section{PROPERTIES OF THE REFLECTORS}

\subsection{Geometry}

Depending on the applied energy at the objects' surfaces (table 3) the reflectors show significant differences regarding their geometry (Figure 7). Eight reflectors were manufactured with varying degrees of energy applied in the surface area (increasing from No. 1 to No. 8). Additionally the laser scanning speed was changed. The energy amount of No. 1 equals that of the samples DP1 and DP2, the amount of No. 8 is between that of the samples DP1 / DP2 and DP3 / DP4.

Inserting a relatively low amount of laser power to the surface of the reflector leads to quite a sharp edge (No. 1, right picture in figure 7). An increased amount of energy leads to a broadened edge. Close to this edge a deformation which surrounds the inner surface of the reflector occurs (No.8). On the other hand the facets of the reflector become much more visible using the increased laser power, expecially without using sand-blasting as finishing method.

Obviously to effects are competitive to each other. On the one hand a low laser power leads to sharp edges of the reflectors outline without producing a parasitic deformation, on the other hand the edges of the facets don't become very clear.

Differing from the test parts in section 4 the reflectors are manufactured on an EOS M290 machine using argon as inert gas. The M290 is nearly identical in construction to the M280.

\subsection{Surface Roughness}

One important point regarding the necessary finishing process is the roughness of the obtained surface. The lower the roughness the smaller the necessary material removal will be. If an indefinite finishing technology like hand-grinding or hand-polishing is used a great amount of material removal will surely lead to a distorted surface geometry. This will have a negative effect on the light distribution of the light module.

The achieved surface-roughness Ra of the untreated pars are in the range of $20-30 \mu \mathrm{m}$. This is comparable to the roughness achieved in a casting process. ${ }^{20}$ The reflector with the highest laser power per area shows a significantly smoother surface.

The typical first step in finishing SLM parts is sand-blasting. This will level surface peaks, remove adhering powder particles and compact the material close to the surface. Nearly all reflectors show an improvement regarding the roughness $\mathrm{Ra}$ and $\mathrm{Rq}$ after sand blasting, except No. 2 and 8. This exceptions are not significant and may also be caused by this measurement comparing the two half sides of the reflector (one half sand-blasted, the other one untreated, see figure 7)

The reflector weight increases with increased application of energy. The difference between the samples No.1 and No.8 makes $9.4 \%$ including the support structure which has not been removed before the measurements. This strong difference is caused by the fact that an increased application of laser power slightly broadens the outline of SLM objects. Since the side faces of the reflector are rather thin (2 $\mathrm{mm}$ and less) this broadening effect can easily be detected by measuring the objects' weight. 
Table 3. Manufacturing parameters and roughness of laser-sintered reflectors

\begin{tabular}{ccccccc}
\hline $\begin{array}{c}\text { Reflector } \\
\text { No. }\end{array}$ & $\begin{array}{c}\text { Laser power per } \\
\text { area (factor) }\end{array}$ & \multicolumn{2}{c}{ Sand-blasted } & \multicolumn{2}{c}{ No finishing } & Reflector \\
& $\mathrm{Ra}(\mu \mathrm{m})$ & $\mathrm{Rq}(\mu \mathrm{m})$ & $\mathrm{Ra}(\mu \mathrm{m})$ & $\mathrm{Rq}(\mu \mathrm{m})$ & weight $(\mathrm{g})$ \\
\hline 1 & 1.0 & 13.19 & 16.93 & 16.49 & 21.24 & 48.018 \\
2 & 1.0 & 18.35 & 23.37 & 17.97 & 23.37 & 49.453 \\
3 & 1.9 & 14.35 & 17.95 & 18.45 & 24.34 & 49.750 \\
4 & 2.0 & 14.89 & 18.69 & 18.89 & 23.81 & 50.925 \\
5 & 2.3 & 12.97 & 16.61 & 22.93 & 30.72 & 51.340 \\
6 & 3.2 & 9.40 & 11.87 & 19.43 & 21.60 & 51.409 \\
7 & 3.3 & 12.31 & 15.32 & 17.26 & 21.42 & 52.244 \\
8 & 4.4 & 8.53 & 10.63 & 7.12 & 9.79 & 52.541 \\
\hline
\end{tabular}

\subsection{Finishing}

For both the milled reflector and the SLM version, further surface finishing steps are required. In order to keep the additional effort as small as possible the investigated SLM reflector is produced using a low value of applied energy at the surface. This gives the best geometrical precision and seems to offer a feasible reflectance value. The parameters are according to that of the samples DP1 and DP2 and of the reflector No.1 from the previous subsections but using a M280 with nitrogen as inert gas.

In both cases (SLM and milling) the threads were cut by hand. The geometry of the mounting slits for the lens was not sufficient at the milled reflector, whereas the SLM reflector was perfect in this case. This fact shall not be taken into account further since the adaption of the CAD- and process parameters will help to avoid this trouble. Some bore holes of the SLM reflector had to be cured by hand meaning nearly no effort.

The most effort had to be made regarding the reflective surface of the SLM reflector. We decided to use an easy to handle 3-axis milling process for the blacking of the reflector. Therefore the CAD geometry of the reflector was supplied with an offset in z-direction. The CAD/CAM generation for this step and the blacking
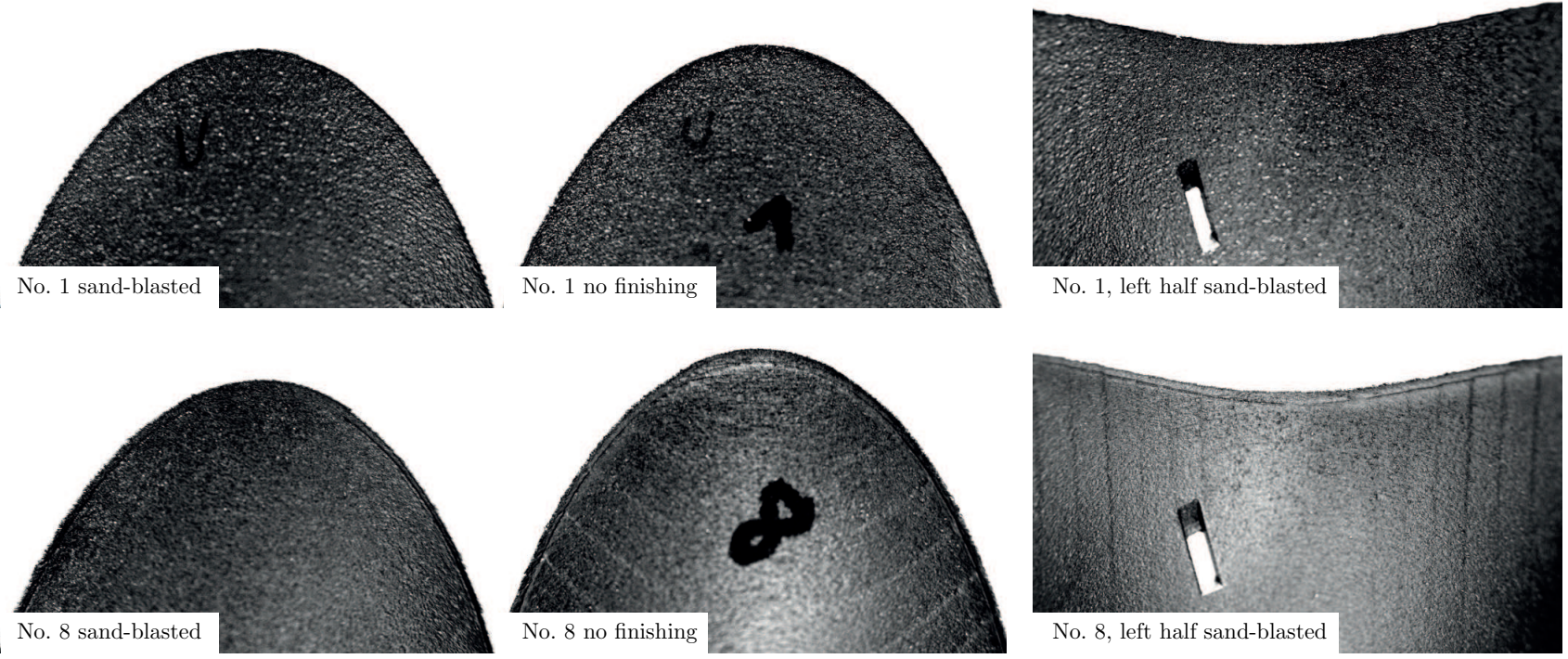

Figure 7. Photos of the laser-sintered reflectors No.1 and 8. 
milling process have to be computed with about $2 \mathrm{~h}$. The hand-polishing needs approximately the same time for both reflectors and the same applies for the assembly of the light module.

\subsection{Manufacturing Time and Costs}

Summing up all the values given in table 1 which excludes the CAD generation of the reflector and the finishing steps, the manufacturing of one milled reflector takes roughly $13 \mathrm{~h}$, whereas $9.5 \mathrm{~h}$ have to be calculated for the SLM one. The finishing of the SLM reflector takes about $2 \mathrm{~h}$ longer than that of the other one. In sum the production of the SLM reflector saves about $1.5 \mathrm{~h}$. On the other hand additional machines are necessary for the SLM process. In our case we used SLM in combination with 3-axis milling in contrast to a pure 5-axis milling process. But the big advantage of the SLM/milling combination is that the milling process only needs to be applied on some functional surfaces, in this case the reflectance surface and the opposite surface connecting reflector and mount. This means only one change of the parts fixation in the milling process. Since this milling process is much easier for the SLM reflector than for the other one, user mistakes are less likely.

While the SLM process saves time in the generation of the reflector, the used material is clearly more expensive. It has to be made clear that the geometry of the reflector is optimized for the 5-axis milling process. An optimization for the SLM process will save some additional time. A parallel production of a small lot of reflectors will reduce the time per sample much more in case of the SLM than of the milled reflector.

\subsection{Light Distribution}

The laser module is made to produce a vertically narrow beam with high intensity at its middle. The prototype with the milled reflector offers a light output of $245 \mathrm{~lm}$ in the range of $\pm 15^{\circ}$ horizontally and $+4 /-6^{\circ}$ vertically. The SLM reflector gives an output of $196 \mathrm{~lm}$. Due to the light output of the lens which overlies that of the
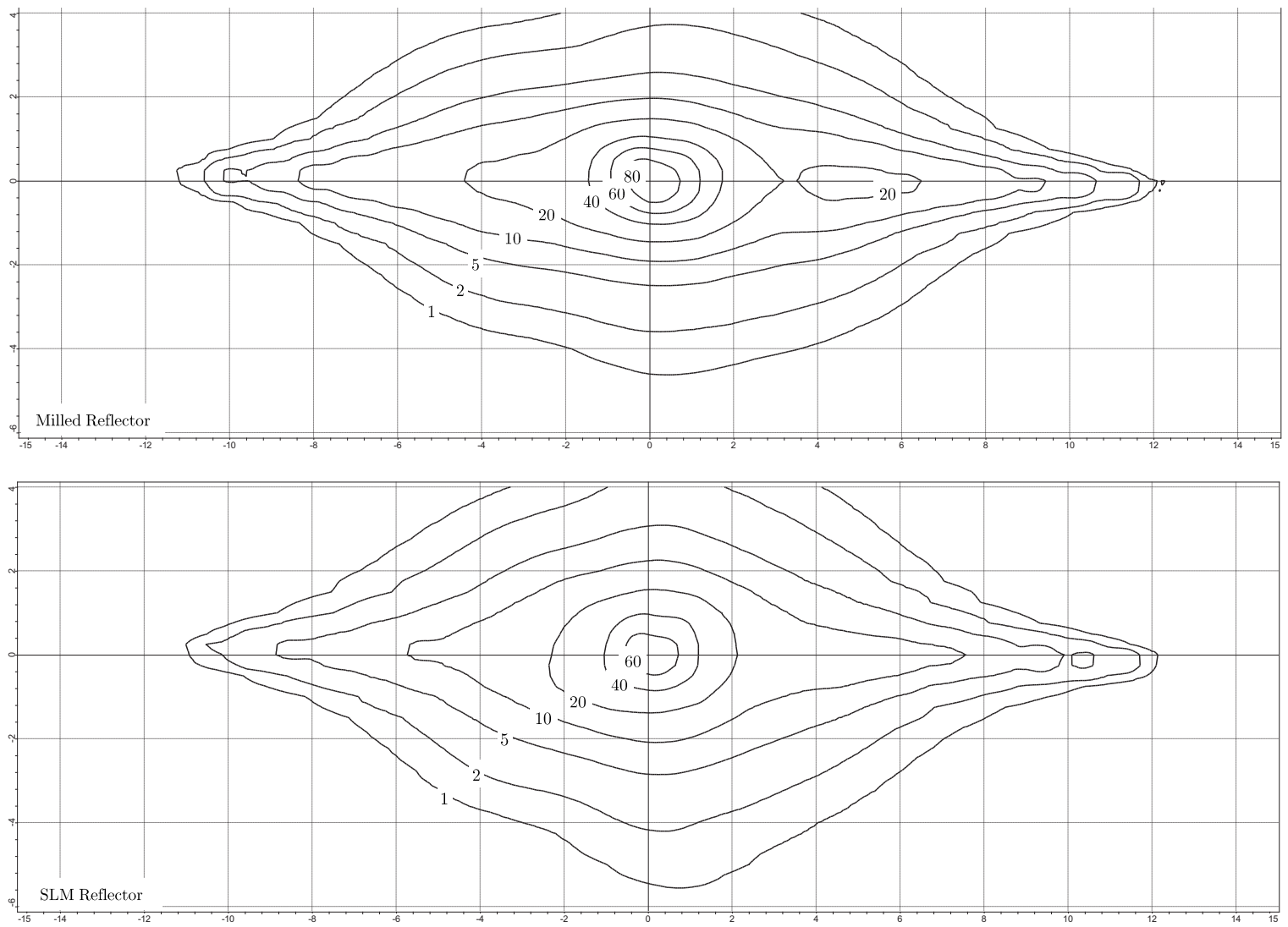

Figure 8. Light distribution of the high beam module with isolux lines. 
reflector these values can't be easily converted into an efficiency of the SLM-reflector. But these results confirm the reduced reflectance of the sintered parts from section 4. This leads to a reduced efficiency of the SLM-based system compared with the conventional one.

The light distribution generated with the two reflectors is given in figure 8 . The slight asymmetry of both distributions is caused by the asymmetric mount of the lens inside of the reflector. The SLM-based light distribution is a bit more extended in vertical direction. This may be caused by a slightly inexact positioning of the phosphor or by inaccuracies which did happen in the milling of the reflector. This indicates that although the milling process for the SLM reflector is much easier, slight deviations in this step may cause noticeable changes in the light distribution. Due to the fact that the secondary mirrors had to be aligned separately for both setups this may cause the vertical broadening as well. The light intensity in the spot is mainly affected by the lens. This indicated a slight difference in the systems setup in both cases. In general the light distribution of both systems is appropriate to evaluate the prototyping modules.

\section{CONCLUSIONS AND OUTLOOK}

The selective laser melting process is used as rapid prototyping technology to manufacture optical reflectors, investigating the surface roughness as well as the reflectance. The required surface properties can be achieved by adding appropriate blacking processes on all relevant faces. Due to the fact that not all faces have to be finished some effort can be saved compared to milling as manufacturing technology. In the investigated case the SLM process will save time but the price of the used material is higher. The SLM process is not only worthwhile for geometrically complex structures that cannot be produced with other technologies but also for parts like the reflector because it simplifies the manufacturing process (in this case the milling process) a lot. Both process chains are suitable to preserve prototypes of optical reflectors.

In order to achieve a precise surface geometry in the SLM process the application of energy in this region has to be kept quite low. On the other hand this creates comparably rough surfaces. Therefore, it may be helpful to adjust the laser energy according to the required surface properties. For faces requiring smooth surface properties, a higher amount of energy has to be applied in order to possibly save a finishing step, whereas for faces with the need for high geometrical precision (sharp edges, bore holes, etc.), a lower amount of energy should be used.

The reflectance of the used SLM material AlSi10Mg is much lower than that of the aluminum used for the

milled reflector (which is in fact another aluminum alloy). In order to evaluate the influence of the SLM process on the reflectance value additional investigations with different SLM materials are desirable.

\section{REFERENCES}

[1] Ye, S., Xiao, F., Pan, Y. X., Ma, Y. Y., and Zhang, Q. Y., "Phosphors in phosphor-converted white light-emitting diodes: Recent advances in materials, techniques and properties," Materials Science and Engineering: R: Reports 71(1), 1-34 (2010).

[2] Lachmayer, R., Wolf, A., and Kloppenburg, G., "System efficiency of laser-based white light," Advanced Optical Technologies 3(5-6) (2014).

[3] BMW Group, "BMW Laserlicht geht in Serie. Der BMW i8 ist das erste Serienfahrzeug mit der innovativen Lichttechnologie." https://www.press.bmwgroup.com/deutschland/pressDetail.html? title=bmw-laserlicht-geht-in-serie-der-bmw-i8-ist-das-erste-serienfahrzeug-mit-derinnovativen\&outputChannelId=7\&id=T0165849DE\&left_menu_item=node__8610 (2014). accessed on 2015-01-05.

[4] AUDI AG, "Weltpremiere auf der CES 2014 in Las Vegas: Der Audi Sport quattro laserlight concept." https://www .audi-mediaservices.com/publish/ms/content/de/public/pressemitteilungen/ 2014/01/02/weltpremiere_auf_der.html (2014). accessed on 2015-01-05.

[5] Pudenz, K., "Mercedes-Benz Concept GLA bekommt Scheinwerfer mit Laserlicht." http://www . springerprofessional.de/mercedes-benz-concept-gla-bekommt-scheinwerfer-mit-laserlicht/ 4379740.html (2014). accessed on 2015-01-05.

[6] Lachmayer, R., Kloppenburg, G., and Stephan, S., "Temperature issues with white laser diodes, calculation and approach for new packages," Proceedings of SPIE 9450 (9450-72) (2014). 
[7] Herbold, C. and Neumann, C., "Vorbild Natur: Bionische Strukturen zur Entwärmung von LEDs," in [Licht 2012], Deutsche Lichttechnische Gesellschaft, ed., 327-334 (2012).

[8] Herbold, C., Herrmann, F., and Neumann, C., "Effiziente Innenleuchten: Von der Wissenschaft in die Produktion," in [Licht 2014], Nederlandse Stichting Voor Verlichtungskunde, ed., 146-151 (2014).

[9] EOS GmbH, "Materialdatenblatt EOS Aluminium AlSi10Mg." http://ip-saas-eos-cms.s3.amazonaws . com/public/2bbfdf00b45afe95/1199a6b59c4568f2186a6c0ca2a7e2e2/E0S_Aluminium_AlSi10Mg_de. pdf (2014). accessed on 2015-01-13.

[10] EOS GmbH, "Materialdatenblatt EOS MaragingSteel MS1." http://ip-saas-eos-cms.s3.amazonaws . com/public/b81bf931642865f9/ddf079c7dc22b4f7601d2035a42574b9/EOS_MaragingSteel_MS1_de.pdf (2011). accessed on 2015-01-13.

[11] Lachmayer, R., Wolf, A., and Kloppenburg, G., "Lichtmodule auf Basis von laseraktiviertem Leuchtstoff für den Einsatz als Zusatzfernlicht," in [6. VDI-Tagung Optische Technologien in der Fahrzeugtechnik // Optische Technologien in der Fahrzeugtechnik], VDI-Berichte 2221, 31-44, VDI-Verl., Düsseldorf (2014).

[12] UN/ECE, "Regulation 113: Uniform provisions concerning the approval of motor vehicle headlamps emitting a symmetrical passing beam or a driving beam or both and equipped with filament, gas-discharge light sources or LED modules," (2012).

[13] Leroux, A., Reiss, B., and Thabet, Z., "Laser Spot: specification study for improved safety and comfort," in [Proceedings of the SIA 2014], Société des Ingénieurs de l'Automobile, ed. (2014).

[14] Lachmayer, R., Wolf, A., Danov, R., and Kloppenburg, G., "Reflektorbasierte Laser-Lichtmodule als Zusatzfernlicht für die Fahrzeugbeleuchtung," in [Licht 2014], Nederlandse Stichting Voor Verlichtungskunde, ed., 16-24 (2014).

[15] Lakshmanan, A., Kumar, R. S., Sivakumar, V., Thomas, P. C., and Jose, M. T., "Synthesis, photoluminescense and thermal quenching of YAG:Ce phosphor for white light emitting diodes," Indian Journal of Pure ESApplied Physics 49, 303-307 (2011).

[16] Adam, G. A. and Zimmer, D., "Design for Additive Manufacturing - Element transitions and aggregated structures," CIRP Journal of Manufacturing Science and Technology 7(1), 20-28 (2014).

[17] Zimmer, D. and Adam, G., "Konstruktionsregeln für Additive Fertigungsverfahren - Design Rules for Additive Manufacturing," Konstruktion (7-8), 77-82 (2013).

[18] Elsbrock, S. S., "Design for Additive Manufacturing - Designing for the process," Proceedings of Dimensional Accuracy and Surface Finish in Additive Manufacturing: ASPE Spring Topical Meeting, 209-213 (2014).

[19] Naumann, H. and Schröder, G., [Bauelemente der Optik], Carl Hanser Verlag, 6 ed. (1992).

[20] "DIN4766 Herstellverfahren der Rauheit von Oberflächen - Surface roughness associated with types of manufacturing method," (1981). 